\title{
A REMARK ON AN EXAMPLE OF R. A. JOHNSON
}

\author{
GEORGE R. SELL ${ }^{1}$
}

\begin{abstract}
In [3] Johnson constructs an example of a second-order linear differential equation with almost periodic coefficients and with an almost automorphic behavior which we describe as Property J. In this paper we give a necessary and sufficient condition that a second order linear differential equation has Property $\mathrm{J}$.
\end{abstract}

In [3] Johnson gives an example of a linear differential equation $x^{\prime}=A(t) x$ where $x \in R^{2}$,

$$
A(t)=\left(\begin{array}{cc}
a(t) & b(t) \\
0 & -a(t)
\end{array}\right)
$$

for suitable almost periodic functions $a(t)$ and $b(t)$, and such that the induced flow in the projective bundle $P S \times H(A)$, where $H(A)$ is the hull of $A$, has precisely two minimal sets $M_{1}$ and $M_{2}$. Moreover, one set $M_{1}$ is an almost periodic minimal set and the other set $M_{2}$ is an almost automorphic extension of $H(A)$ that is not almost periodic. The existence of second-order linear differential equations with almost periodic coefficients and with this almost automorphic behavior in $P S \times H(A)$ was predicted in [2]. Since this phenomenon is important for the classification of such equations, we make the following definition:

A linear differential equation $x^{\prime}=A(t) x, x \in R^{2}$, is said to have Property $\mathrm{J}$ if $A(t)$ has almost periodic coefficients and there are two minimal sets $M_{1}$ and $M_{2}$ in the induced projective flow on $P S \times H(A)$ where $M_{1}$ is an almost periodic minimal set and $M_{2}$ is an almost automorphic extension of $H(A)$ that is not almost periodic.

The purpose of this note is to derive a necessary and sufficient condition for

$$
x^{\prime}=A(t) x, \quad x \in R^{2},
$$

to have Property J. Before stating our result recall that the mean value of any almost periodic function $a(t)$ is given by

$$
M(a)=\lim _{T \rightarrow \infty} \frac{1}{T} \int_{0}^{T} a(t) d t .
$$

Theorem. A necessary and sufficient condition for equation (2) to have Property J is that there is an almost periodic Lyapunov-Perron transformation $x=P(t) y$ (i.e. $P, P^{-1}$ and $\dot{P}$ are almost periodic in $\left.t\right)$ such that $B=P^{-1}(A P-\dot{P})$ is upper

Received by the editors May 22, 1980.

1980 Mathematics Subject Classification. Primary 34C27.

Key words and phrases. Almost automorphic, almost periodic, linear differential equations.

${ }^{1}$ This research was supported in part by NSF Grant MCS 79-01998. 
triangular and almost periodic in $t$, say

$$
B(t)=\left(\begin{array}{cc}
u(t) & v(t) \\
0 & w(t)
\end{array}\right)
$$

and the associated inhomogeneous equation

$$
\xi^{\prime}=(u-w) \xi+v
$$

has a bounded solution that is not almost periodic in $t$. In this case, the following properties are valid:

(i) For some functions $\left(u^{*}, v^{*}, w^{*}\right)$ in the hull $H(u, v, w)$ the equation $\xi^{\prime}=$ $\left(u^{*}-w^{*}\right) \xi+v^{*}$ has a bounded almost automorphic solution that is not almost periodic.

(ii) $M(u)=M(w)=\frac{1}{2} M(\operatorname{tr} A)$.

(iii) The integral $\int_{0}^{t}[u(s)-w(s)] d s$ is unbounded in $t$.

(iv) At least one of the integrals $\int_{0}^{t}[u(s)-M(u)] d s, \int_{0}^{t}[w(s)-M(w)] d s$ is unbounded in $t$.

(v) $v(t) \neq 0$.

Proof. In order to prove this theorem we shall use the fact that if (2) has Property $\mathrm{J}$ and if $x=P(t) y$ is any almost periodic Lyapunov-Perron transformation then

$$
y^{\prime}=B(t) y
$$

has Property $\mathrm{J}$ where $B=P^{-1}(A P-\dot{P})$, cf. [2].

Now assume that (2) has Property J. Then the almost periodic minimal set $M_{1}$ must be an $N$-fold cover of $H(A)$. (In fact, it is a 1 -fold cover of $H(A)$.) The almost periodic Lyapunov-Perron transformation $x=P(t) y$ that reduces (2) to (5), where $B(t)$ is given by (3), is assured by [5, Theorem 9]. Next let $(r, \theta)$ denote the polar coordinates in the $y$-plane. Since $B(t)$ is upper triangular, the minimal set $M_{1}$ for (5) is generated by $\theta=0$ (or $\pi$ ). The other minimal set $M_{2}$ must then be bounded away from 0 and $\pi$. This means that if $\theta(t)$ is the $\theta$-coordinate of the solution of (5) that originates in $M_{2}$, then $\cot \theta(t)$ is bounded in $t$. However, $\xi(t)=\cot \theta(t)$ is necessarily a solution of (4). It is bounded and not almost periodic. On the other hand, if (4) has a bounded solution that is not almost periodic, then by [4, Proposition 3.8] statement (i) is valid. Also the argument used by Johnson [3] shows that (5) has Property $\mathrm{J}$ where $B(t)$ is given by (3).

In order to prove statement (ii) we shall use the properties of the spectrum $\Sigma(A)$ and $\Sigma(B)=\{M(u), M(w)\}$. Since [2] Property $\mathrm{J}$ implies that $\Sigma(A)$ consists of one point (which is necessarily $\left\{\frac{1}{2} M(\operatorname{tr} A)\right\}$ ) statement (ii) now follows. Since (4) has a bounded solution that is not almost periodic and since $M(u-w)=0$, it follows from Favard's Theorem [1, pp. 101, 107] that statement (iii) is valid. Statement (iv) now follows immediately from (ii) and (iii). If $v(t) \equiv 0$, then it follows that $\theta=\pi / 2$ generates an almost periodic minimal set $M_{3}$ in the induced projective flow on $P S \times H(A)$. In other words there are three distinct minimal sets $\left\{M_{1}, M_{2}, M_{3}\right\}$ in this flow. Consequently by [6, Theorem 8] the induced flow on $P S \times H(A)$ is distal. Since the restriction of this flow to $M_{2}$ is not distal, we have a contradiction. Q.E.D. 
REMARK. We cannot conclude, as in Johnson's example, that $u=-w$ in (3). However if $x^{\prime}=A(t) x, x \in R^{2}$, is given with almost periodic coefficients, then for any almost periodic function $\alpha(t)$ the shifted equation

$$
x^{\prime}=(A(t)-\alpha(t) I) x
$$

induces the same flow on $P S \times H(A)$, cf. [5, p. 29]. Furthermore, if $x=P(t) y$ transforms (2) to (5), then this will change (6) to $y^{\prime}=(B(t)-\alpha(t) I) y$. Consequently if one chooses $\alpha(t)=\frac{1}{2} \operatorname{tr} A(t)$, then the upper triangular matrix $(B-\alpha I)$ has the form (1).

\section{REFERENCES}

1. A. M. Fink, Almast periodic differential equations, Lecture Notes in Math., vol. 377, Springer-Verlag, New York, 1974.

2. R. A. Johnson, On a Floquet theory for almost periodic two-dimensional linear system, J. Differential Equations 37 (1980), 184-205.

3. A linear, almost periodic equation with an almost automorphic solution, Proc. Amer. Math. Soc. 82 (1981), 199-205.

4. __ Bounded solutions of scalar, almost periodic linear equations, Illinois J. Math. (to appear).

5. R. J. Sacker and G. R. Sell, Lifting properties in skew-product flows with applications to differential equations, Mem. Amer. Math. Soc. No. 190, 1977.

6. __ A spectral theory for linear differential systems, J. Differential Equations 27 (1978), 320-358.

School of Mathematics, University of Minnesota, Minneapous, Minnesota 55455

Current address: Department of Mathematics, University of Southern California, Los Angeles, California 90007 